\title{
Incidental Diagnosis of a Carcinoid Tumor of the Ileum using Contrast-Enhanced Ultrasound (CEUS)
}

\section{Introduction}

Carcinoids of the gastrointestinal system are a rare manifestation of highly vascularized tumors that arise from enterochromaffin cells with a low incidence rate of approx. 2/100000 people and a mean age of diagnosis in the 6th life decade (Crocetti E, Paci E. Eur J Cancer Prev 2003;12:191-4). About $42 \%$ of all gastrointestinal carcinoids occur in the small bowel, followed by carcinoids of the appendix (about 23\%) and of the stomach (about $9 \%$ ) (Modlin IM et al. Cancer 2003;97: 934-59). Tumors greater than $2 \mathrm{~cm}$ in size normally show classic clinical signs and symptoms and tend to develop metastases. Typical clinical symptoms include abdominal cramping, diarrhea and flushing (Caplin ME et al. Lancet 1998;352:799-805). Because of the low clinical incidence and the small tumor size, initial diagnosis of this tumor entity is difficult. Diagnostic options include all available imaging modalities, including (contrast-enhanced) ultrasound (CEUS) and color Doppler, as well as (PET) computed tomography and magnetic resonance imaging. With the broader availability of CEUS in the clinical routine and improved imaging quality in recent years, ultrasound has the potential to be a viable imaging option for the evaluation of suspicious lesions in the terminal ileum (Rickes $S$ et al. Med Klin (Munich). 2009;104(7):564-6.)

\section{Case Report}

A 52-year-old man presented with diarrhea and abdominal cramping. The initial physical examination revealed no palpable pathologies of the abdomen and initial native B-mode/color Doppler ultrasound of the abdomen was recommended by the referring physician. This initial ultrasound examination was performed using a 1-7 MHz multifrequency curved-array transducer with color Doppler and contrast-enhanced ultrasound capabilities. The used diagnostic device (RS80A with Prestige, Samsung Medison Co., Ltd., Seoul, Korea) had the capability to save and store dynamic image sequences as cine loops. SonoVue (Bracco, Milan, Italy) was used as the ultrasound contrast agent. Extensive initial B-mode ultrasound revealed a $2 \mathrm{~cm}$, solid hypoechoic lesion inside the wall of the small bowel in the right lower abdomen, additionally showing major vascularization using color Doppler. After the single injection of $2.4 \mathrm{ml}$ of SonoVue followed by a flush of $10 \mathrm{ml}$ of a $0.9 \mathrm{NaCl}$ solution, CEUS revealed a highly arterial hypervascularized lesion inside the wall of the terminal ileum, highly suspicious of a tumor and in line with sonographic features of a carcinoid tumor of the ileum (• Fig. 1). The tumor showed persistent hypervascularization during the late phase, additionally revealing necrotic areas. Additional blood tests showed an elevated serotonin blood level $(436 \mathrm{ng} / \mathrm{ml}$; reference $<193 \mathrm{ng} / \mathrm{ml}$ ) and an additional $\mathrm{Ga}$ DOTA-TATE PET-CT scan was recommended for staging of metastases. The PET-CT scan revealed no metastases and showed a somatostatin receptor (SSR)-positive tumor in the terminal ileum and surgical resection of the tumor was recommended. We intraoperatively evaluated the perfusion of the tumor again using a high-resolution linear probe (L9-2) using color Doppler and CEUS
( $\triangleright$ Fig. 2,3 ) directly guiding the surgeon to the suspicious lesion for resection. Histopathological workup confirmed the initial diagnosis of a carcinoid tumor of the ileum ( Fig. 4). After resection, the patient recovered quickly and was able to be discharged without complications.

\section{Discussion}

Sonographic characterization and imaging of tumors of the gastrointestinal system is challenging with respect to the difficulties evaluating gastrointestinal structures. The terminal ileum is sonographically rather easy to evaluate but imaging options for the evaluation of lesions as well as the spatial resolution of some ultrasound systems were limited in the past. For a long time, ultrasound was considered to be not particularly useful for evaluation of the terminal ileum. The largest study conducted so far evaluating carcinoids using CEUS examined 33 primary lesions and found similar perfusion characteristics as described in this case report (Dorffel Y, Wermke W. Ultraschall in der Medizin. 2008;29(5):506-14) with

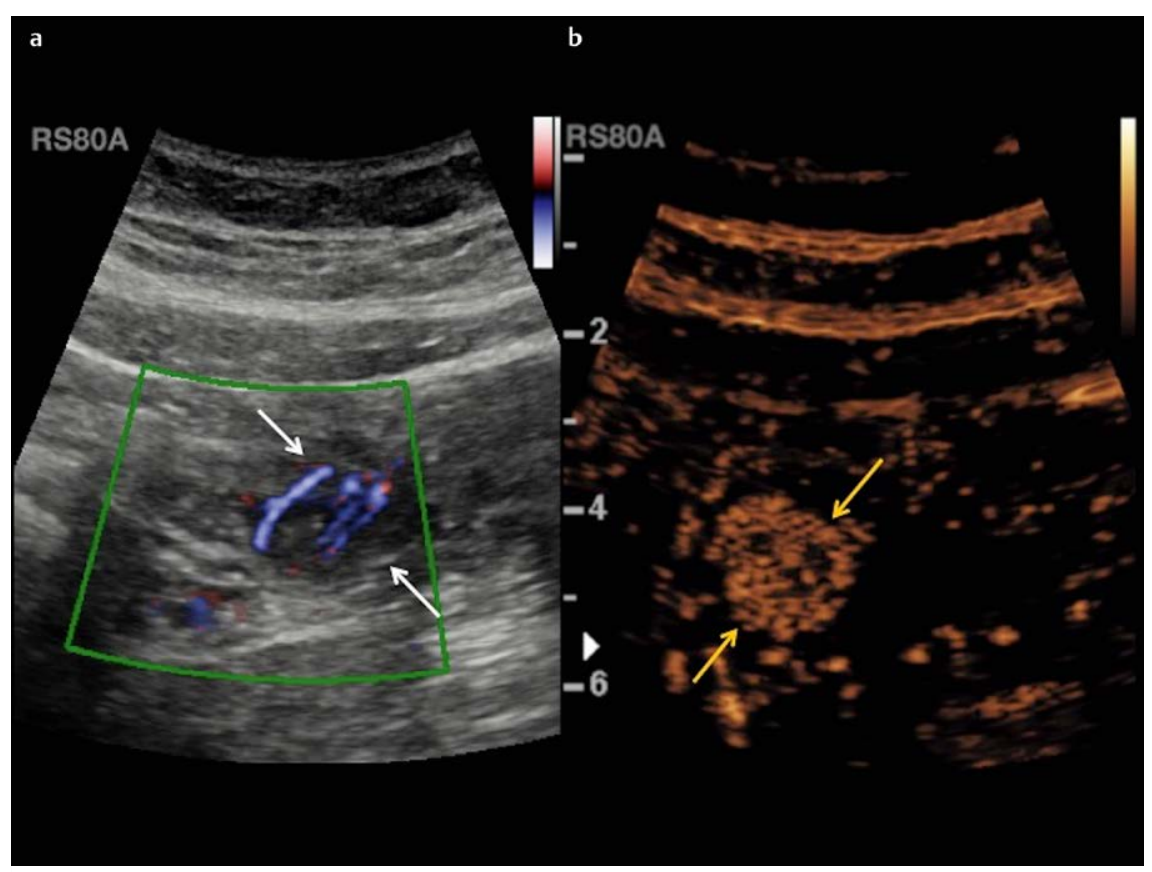

- Fig. 1 Color Doppler ultrasound a and contrast-enhanced ultrasound $\mathbf{b}$ of a suspicious hypoechoic mass in the small bowels of the right lower abdomen shows major vascularization in color Doppler mode (white arrows) as well as major hypervascularization in the arterial phase (yellow arrows) suspicious of a carcinoid of the terminal ileum. 


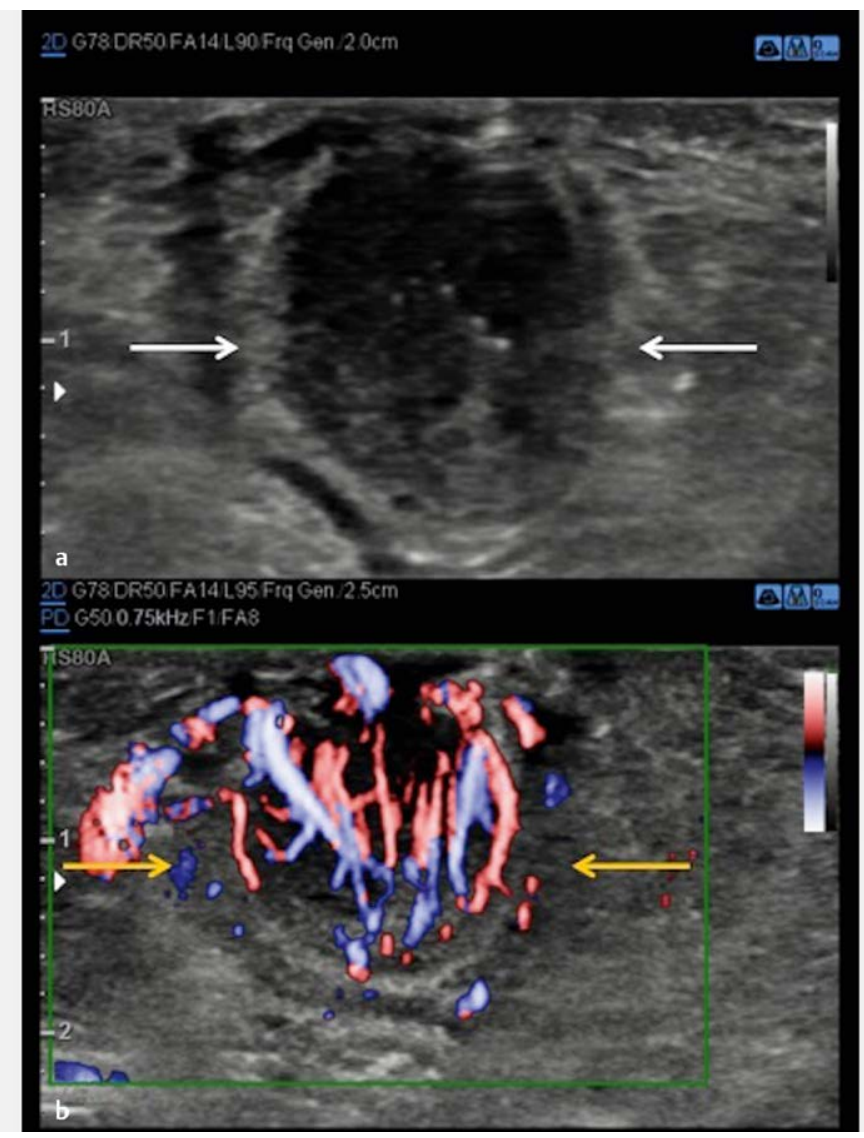

- Fig. 2 Additional intraoperative B-mode ultrasound a and color Doppler a using a high-definition linear probe (L9-2). The lesion can clearly be visualized using B-mode ultrasound (white arrows) and vascularization can be clearly visualized using color Doppler mode (yellow arrows).

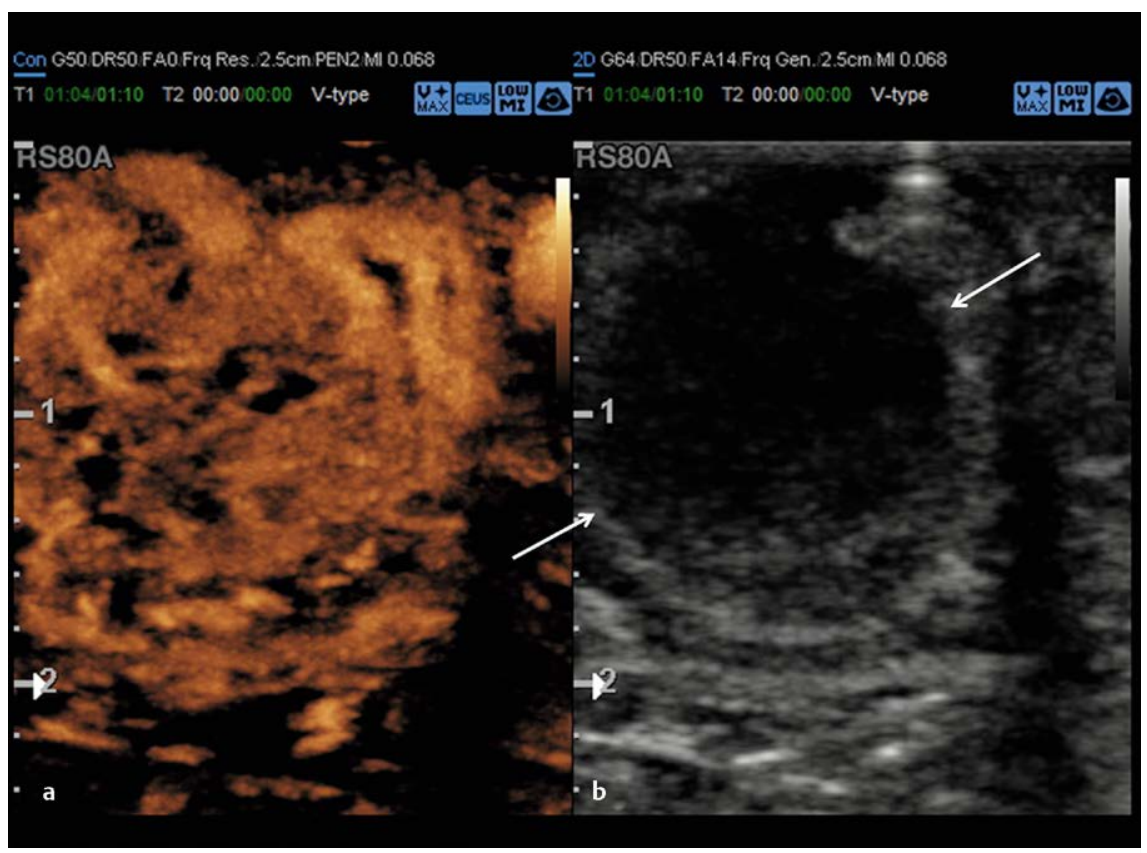

- Fig. 3 Additional intraoperative side-by-side contrast-enhanced ultrasound $\mathbf{a}$ and B-mode ultrasound $\mathbf{b}$ using the same high-definition linear probe (L9-2). The lesion can be clearly visualized using B-mode ultrasound (white arrows) and vascularization patterns as well as lesion boundaries can be evaluated using the contrast mode of the ultrasound system guiding the surgeons to the lesion. strong arterial hypervascularization. CEUS makes it possible to visualize the characteristic perfusion patterns of carcinoids with strong arterial hypervascularization, thus facilitating diagnosis and adding diagnostic confidence, especially in comparison to color Doppler. Additional benefits of this examination technique include the ability to evaluate and characterize unclear lesions of the terminal ileum in the same ultrasound session and the use of an almost side effect-free contrast agent that is not dependent on thyroid and renal function, thus making it a viable and cost-effective technique without ionizing radiation compared to the other imaging modalities in the daily clinical routine.

\section{Conclusion}

We describe the diagnosis of a carcinoid of the terminal ileum using CEUS. CEUS made it possible to characterize the lesion by showing the typical hypervascularization of these tumors and by evaluating the dynamic enhancement pattern during the arterial and venous phase. Additional intraoperative ultrasound guided the surgeons to the correct location of the tumor and added diagnostic confidence for resection of the tumor. CEUS was able to sufficiently evaluate tumor perfusion, and, with modern ultrasound and imaging techniques, CEUS seems to be a viable option for the characterization of these tumors in the terminal ileum with additional clinical benefits.

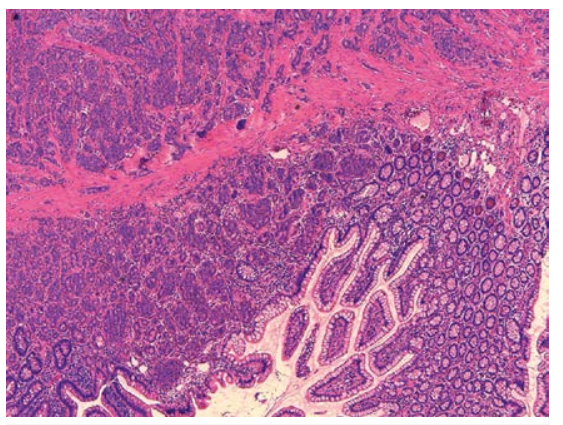

- Fig. 4 Histological workup (hematoxylin and eosin stain) of the suspicious lesion after surgical resection shows atypical tumor cells with good differentiation arranged in a typical Zellballen pattern with infiltration of the lamina muscularis mucosae in line with the histological patterns of a carcinoid of the ileum. 


\section{Catchphrases}

Contrast-enhanced ultrasound - carcinoid tumor - dynamic perfusion

\section{Conflict of Interest}

The authors have no conflict of interest to disclose.

Authors

Johannes Rübenthaler ${ }^{1}$, Karolin Paprottka ${ }^{1}$, Bernhard Renz², Markus Rentsch², Thomas Knösel ${ }^{3}$, Maximilian Reiser ${ }^{1}$, Dirk-André

Clevert $^{1}$

\section{Affiliations}

1 Department of Clinical Radiology, Ludwig-Maximilians-University of MunichGrosshadern Campus, Munich, Germany

2 Department of General, Visceral, Transplantation Surgery, LMU Munich, Munich, Germany

3 Department of Pathology, LMU Munich, Munich, Germany
Bibliografie

Dr. Johannes Rübenthaler, MD

Ludwig-Maximilians-University of Munich-Grosshadern Campus

Department of Clinical Radiology

Marchioninistr. 15

81377, Munich

Germany

Tel: 004989440073627,

Johannes.Ruebenthaler@med.uni-muenchen.de
DOI https://doi.org/10.1055/s-0043-110474

Ultrasound Int Open 2017; 3: E122-E124

(c) Georg Thieme Verlag KG Stuttgart .

New York

ISSN 2199-7152

\section{(c) (i) ()ㅜ $€$}

